\title{
Influence of shear effects on the characteristics of axisymmetric wave propagation in a buried fluid- filled pipe
}

\section{Ping Lu (D277717386@qq.com )}

Southwest Jiaotong University https://orcid.org/0000-0002-2661-2094

\section{Xiaozhen Sheng}

Shanghai University of Engineering Science

\section{Yan Gao}

Chinese Academy of Sciences

\section{Ruichen Wang}

University of Huddersfield

\section{Original Article}

Keywords: Buried fluid-filled pipe, Axisymmetric waves, Shear effect, Dispersion

Posted Date: October 30th, 2020

DOI: https://doi.org/10.21203/rs.3.rs-44967/v2

License: (a) (i) This work is licensed under a Creative Commons Attribution 4.0 International License. Read Full License 


\section{Influence of shear effects on the characteristics}

\section{of axisymmetric wave propagation in a buried fluid-filled pipe}

Ping Lu

State Key Laboratory of Traction Power, Southwest Jiaotong University, Chengdu 610031, China;

Emai1:277717386@qq.com

Xiaozhen Sheng

School of Urban Railway Transportation, Shanghai University of Engineering Science, Shanghai 201620,

China;

Email: shengxiaozhen@hotmail.com

Yan Gao

Key Laboratory of Noise and Vibration Reasearch, Institute of Acoustics, Chinese Academy of Sciences, Beijing 100190, China;

Email: gaoyan@mail.ioa.ac.cn

Ruichen Wang

Institute of railway research, University of Huddersfield, Huddersfield HD1 3DH, UK.

Email:r.wang@hud.ac.uk

Corresponding author: Ping Lu; Email:277717386@qq.com ; Tel: +86-13540197065 


\title{
Influence of shear effects on the characteristics of axisymmetric wave propagation in a buried fluid-filled pipe
}

\author{
Ping $\mathrm{Lu}^{1}$, Xiaozhen Sheng ${ }^{2}$, Yan Gao ${ }^{3}$, Ruichen Wang ${ }^{4}$
}

1. State Key Laboratory of Traction Power, Southwest Jiaotong University, Chengdu 610031, China;

2. School of Urban Railway Transportation, Shanghai University of Engineering Science, Shanghai 201620, China;

3. Key Laboratory of Noise and Vibration Reasearch, Institute of Acoustics, Chinese Academy of Sciences, Beijing 100190, China;

4. Institute of railway research, University of Huddersfield, Huddersfield HD1 3DH, UK. Corresponding author email:277717386@qq.com ; Tel: +86-13540197065

\begin{abstract}
The acoustic propagation characteristics of axisymmetric waves have been widely used in leak detection of fluid-filled pipes. The related acoustic methods and equipment are gradually coming to the market, but their theoretical research obviously lags behind the field practice, which seriously restricts the breakthrough and innovation of this technology. Based on the fully three-dimensional effect of the surrounding medium, a coupled motion equation of axisymmetric wave of buried liquid-filled pipes is derived in detail, a contact coefficient is used to express the coupling strength between surrounding medium and pipe, then, a general equation of motion was derived which contain the pipe soil lubrication contact, pipe soil compact contact and pipe in water and air. Finally, the corresponding numerical calculation model is established and solved used numerical method. The shear effects of the surrounding medium and the shear effects at the interface between surrounding medium and pipe are discussed in detail. The output indicates that the surrounding medium is to add mass to the pipe wall, but the shear effect is to add stiffness. With the consideration of the contact strength between the pipe and the medium, the additional mass and the pipe wall will resonate at a specific frequency, resulting in a significant increase in the radiation wave to the surrounding medium. The research contents have great guiding effect on the theory of acoustic wave propagation and the engineering application of leak detection technology in the buried pipe.
\end{abstract}

Keywords: Buried fluid-filled pipe; Axisymmetric waves; Shear effect; Dispersion

\section{Introduction}

With the development of urbanisation in past decades, the component like pipe has become a necessary means of liquid and gas transportation. The issue of plumbing leakage is a widespread concern in both industry and academy due to its social, environmental and economic utility. It has been estimated that, in mainland China, current direct economic losses caused by underground pipe network leaks exceed 200 billion Yuan (approximately 22.65 billion pound sterling), sometimes the pipe leakage might cause unexpected major source of hidden danger not only for damaging urban 
biological environment and the security of people's lives and properties. The latest research of the 'track and trace' technology for buried pipelines, which is widely used in the transportation of liquid and gas media such as crude oil and natural gas, is being widely discussed and studied extensively. Acoustic detection methods has attracted more attention because of such non-destructive evaluation does not directly destroy the structure of the original piping system [1-4]. When the leaking of the pipeline happened, the high-pressure fluid in the pipe will be pressured out of the pipeline and cause unavoidable noise, The acoustic leak detection method is used to detect the events of leaks in different locations of the pipeline, using cross-correlation technique to estimate the delay of leakage noise between two measuring points, then the location of leakage would be calculated [5]. The effectiveness of these methods much depends on the rationality of the selection of the propagation characteristic parameters of the dominant wave in the pipeline.

The studies of the acoustic characteristics and propagation mechanism of pipes is developed with pipe leak detection. At present, the acoustic leak detection and location of pipelines are usually carried out by time delay estimation method, which depends strongly on wave propagation characteristics. The early research was mainly to solve the wave equation in thin-walled shell pipe, in which the external medium outside pipes is considered vacuum. Fuller, et.al[6] derived the propagation characteristics of $\mathrm{n}=0$ wave within elastic fluid-filled pipes in vacuo defined as "hard" and "soft" shells, then the energy distribution of radial input force and internal pressure fluctuation under various waveforms is studied theoretically [7]. Xu, et.al[8] studied the vibration propagation characteristics of liquid filling pipes in a vacuum environment. Pinnington, et al[9] established an acoustic propagation model of cast iron pipes without considering the dispersion characteristics, after that, studied the $n=0$ wave propagation characteristic and transfer equation of the pressurised pipes [10,11]. With the development of pipe research, the influence of the medium around the pipes has gradually attracted people's attention. Sinha, et al. [12] studied the numerical results of acoustic wave propagation characteristics of fluid-filled pipes in infinite fluid. Greenspon [13] presented the axisymmetric vibration of thick-wall and thin-walled liquid-filled pipes in water medium. Long et al. [14] put forward a model of acoustic velocity dispersion in the process of acoustic vibration signal propagation and verified by experiments. Zhang, et al. [15] proposed a calculation model for sound velocity under different pipeline embedding conditions. Muggleton et al. [16-18] analysed the propagation characteristics of fluid-dominated axisymmetric waves $(s=1, n=0)$ in filled buried pipes. Gao et al. [19,20] developed a general expression for the fluid-dominated wavenumber in a thin-walled fluid-filled pipe surrounded by a layered elastic soil, and the influence of load effect on elastic medium around pipeline is considered.

Current research studies reveal that at low frequencies, the fluid-dominated axisymmetric wave is not only the main carrying waveform of the vibration energy within the buried fluid-filled pipe, but also is an effective signal component which can be used for pipe leakage inspection. This waveform corresponds to the breathing mode of the pipeline, and the 
current researches on the problem are mainly focused on the metal pipeline. Due to the flexibility of the plastic pipe, the coupling between the pipe and the surrounding medium (mainly soil) is significant, making the influence of the acoustic wave propagation speed and the damping characteristics of the surrounding medium on the energy attenuation more complicated. However, such coupling effect has not been properly addressed in the past; especially the actual contact strength of the pipe-medium interface cannot be considered. With the large-scale use of plastic pipes and the frequent leakage hazards in China's urbanisation construction, it is urgent to carry out related research to avoid unnecessary costs.

In this paper, the coupling vibration equation of "soil-pipe-fluid" is derived in detail, the acoustic wave propagation characteristic model of the buried pipeline is established, and the shear effect of the medium outside the pipe and the shear effect of the interface between the pipe and the medium on the axisymmetric wave of the fluid dominant are discussed.

\section{Differential equations of motion of the medium-pipe-fluid system}

\subsection{Free Motion Equation of fluid-Filled Pipe}

In this section, the coupled motion equations of fluid dominant axisymmetric waves in a buried fluid-filled pipes are deduced based on the motion equations of the fluid-filled pipe in vacuum [6]. The soil medium around the pipes is regarded as a homogeneous and isotropic elastic medium which allows both compression wave and shear wave to propagate. According to the current research, the $s=1$ wave is usually the main carrier of energy in the leakage signal therefore of most interest, and the dynamic damping effect of the pipeline is neglected. Fig.1 shows the cylindrical coordinates of pipes, where $u, v, w$ are the shell displacements in the $\operatorname{axial}(x), \operatorname{circumferential}(\theta)$, and $\operatorname{radial}(r) \operatorname{directions}$, respectively. $a$ and $h$ are the pipe radius and the wall thickness respectively and is assumed $h / a \square 1$. The internal fluid is assumed to be inviscid, and both the surrounding medium and internal fluid are assumed to be lossless.

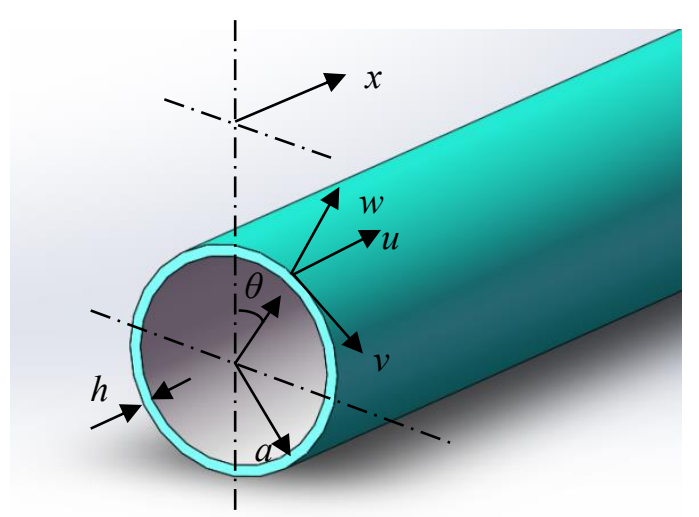

Fig.1 The coordinate system for a buried fluid-filled pipe

For axisymmetric waves $(n=0)$, the rotational motion of the pipe can be neglected, so the circumferential 
displacement and shear stress are both set to zero, free-motion equation of fluid-filled pipe can be described simply according to Donnell-Mushtari shell equation [21] as follow

$$
\left[\begin{array}{ll}
A_{11} & A_{13} \\
A_{31} & A_{33}
\end{array}\right]\left(\begin{array}{l}
u \\
w
\end{array}\right)=\frac{1-v_{p}^{2}}{E h}\left(\begin{array}{c}
0 \\
p_{f}(a)
\end{array}\right)
$$

where $A_{11}=\frac{\partial^{2}}{\partial x^{2}}-\frac{\rho_{p}\left(1-v_{p}^{2}\right)}{E_{p}} \frac{\partial^{2}}{\partial t^{2}} ; \quad A_{13}=\frac{v_{p}}{a} \frac{\partial}{\partial x} ; \quad A_{13}=A_{31} ; \quad A_{33}=\frac{1}{a^{2}}+\delta^{2} a^{2} \frac{\partial^{4}}{\partial x^{4}}+\frac{\rho_{p}\left(1-v_{p}^{2}\right)}{E_{p}} \frac{\partial^{2}}{\partial t^{2}} ;$

Here, $\rho_{p}, E_{p}, v_{p}$ are the density, Young's modulus and Poisson's ratio of the pipe; $\delta$ is the stiffness factor, $\delta^{2}=h^{2} / 12 a^{2} ; \quad p_{f}(a)$ is the internal pressure at the fluid pipe interface.

\subsection{Motion Equation of Soil Medium}

The displacement of soil medium in all directions can be present as $\mathbf{u}(x, r, \theta, t)$. For axisymmetric motion, the torsional displacement can be ignored, then $u_{\theta}=0$. Travelling wave solutions for the surrounding medium may be assumed of the form:

$$
\left\{\begin{array}{l}
u_{x}=U_{m} \mathrm{e}^{\mathrm{i}\left(\omega t-k_{n s} x\right)} \\
u_{r}=W_{m} \mathrm{e}^{\mathrm{i}\left(\omega t-k_{n s} x\right)}
\end{array}\right.
$$

Where $W_{m}, U_{m}$ are the amplitude in radial and axial motion of soil medium, which are the functions of radius, $r$. In the column coordinate system, the expansion process of soil medium can be expressed as

$$
\Delta=\frac{1}{r} \frac{\partial}{\partial r}\left(r u_{r}\right)+\frac{\partial u_{x}}{\partial x}
$$

Here, $\partial / \partial_{\theta}=0$. So, the rotating components of soil medium in the axial and radial direction can also be ignored. The rotating component in the direction $\theta$ is

$$
\varpi_{\theta}=\frac{1}{2}\left(\frac{\partial u_{r}}{\partial x}-\frac{\partial u_{x}}{\partial r}\right)
$$

Substituting for $u_{r}$ and $u_{x}$ from Eq. (2) into Eq. (3) and Eq. (4) gives

$$
\left\{\begin{array}{l}
\Delta=\left(\frac{\partial W_{m}}{\partial r}+\frac{W_{m}}{r}-i k_{s} U_{m}\right) \exp \left(\mathrm{i}\left(\omega t-k_{s} x\right)\right) \\
\varpi_{\theta}=\frac{1}{2}\left(-\mathrm{i} k_{s} W_{m}-\frac{\partial U_{m}}{\partial r}\right) \exp \left(\mathrm{i}\left(w t-k_{s} x\right)\right)
\end{array}\right.
$$

According to stresses waves in solids [22], the displacement of a point in the soil medium satisfies the equation of motion

$$
\left(\lambda_{m}+\mu_{m}\right) \nabla(\Delta)+\mu_{m} \nabla^{2} \mathbf{u}=\rho_{m} \frac{\partial^{2} \mathbf{u}}{\partial t^{2}}
$$

Where $\lambda_{m}, \mu_{m}$ are Lame coefficients; $\rho_{m}$ is the density of the medium; $\nabla$ is the Hamilton differential operator.

The motion equation of soil medium can be obtained by combining Eq. (2)-Eq. (6) 


$$
\left\{\begin{array}{l}
\frac{\partial^{2} \Delta}{\partial r^{2}}+\frac{1}{r} \frac{\partial \Delta}{\partial r}+\left(k_{d s}^{r}\right)^{2} \Delta=0 \\
\frac{\partial^{2} \varpi_{\theta}}{\partial r^{2}}+\frac{1}{r} \frac{\partial \varpi_{\theta}}{\partial_{r}}-\frac{\varpi_{\theta}}{r^{2}}+\left(k_{r s}^{r}\right)^{2} \varpi_{\theta}=0
\end{array}\right.
$$

Where $k_{d s}^{r}, k_{r s}^{r}$ are the compression and shear wavenumbers of soil in radial direction respectively, which can be expressed by compressed wavenumber $k_{d}$, Shear Wavenumber $k_{r}$ and wavenumber in axil direction $k_{s}$ as follow

$$
\left(k_{d s}^{r}\right)^{2}=k_{d}^{2}-k_{s}^{2} ;\left(k_{r s}^{r}\right)^{2}=k_{r}^{2}-k_{s}^{2}
$$

Here, $k_{d}^{2}=\rho_{m} \omega^{2} /\left(\lambda_{m}+2 \mu_{m}\right) ; k_{r}^{2}=\rho_{m} \omega^{2} / \mu_{m}$.

Equation of motion shown in Eq. (7) can be expressed as the Bessel equation of cylindrical space outside the pipe

$$
\left\{\begin{array}{c}
\Delta=G H_{0}\left(k_{d s}^{r} r\right) \\
\varpi_{\theta}=H H_{1}\left(k_{r s}^{r} r\right)
\end{array}\right.
$$

Where $G, H$ are the functions of coordinate in the axial $(x)$ and time $(t) . H_{0}(), H_{1}()$ are the Hankel functions of the second kind which describe outgoing waves.

In order to satisfy Eqs. (5) and (9), $U_{m}, W_{m}$ must have the form [22]

$$
\left\{\begin{array}{l}
U_{m}=-\mathrm{i} A_{m} k_{s} H_{0}\left(k_{d s}^{r} r\right)+\mathrm{i} B_{m} \frac{1}{r} \frac{\partial}{\partial r}\left[r H_{1}\left(k_{r s}^{r} r\right)\right] \\
W_{m}=A_{m} \frac{\partial}{\partial r}\left[H_{0}\left(k_{d s}^{r} r\right)\right]-B_{m} k_{s} H_{1}\left(k_{r s}^{r} r\right)
\end{array}\right.
$$

Where $A_{m}, B_{m}$ are constants.

Substituting Eq. (2) into Eq. (10) gives

$$
\left(\begin{array}{l}
u_{x} \\
u_{r}
\end{array}\right)=\mathbf{T}_{1}\left(\begin{array}{l}
A_{m} \\
B_{m}
\end{array}\right) \mathrm{e}^{\mathrm{i}\left(\omega t-k_{s} x\right)}
$$

Where $\mathbf{T}_{1}=\left[\begin{array}{cc}-\mathrm{i} k_{s} \mathrm{H}_{0}\left(k_{d s}^{r} r\right) & \mathrm{i}\left(k_{r s}^{r}\right)^{2} \mathrm{H}_{0}\left(k_{r s}^{r} r\right) \\ k_{d s}^{r} \mathrm{H}_{0}{ }^{\prime}\left(k_{d s}^{r} r\right) & k_{s} \mathrm{H}_{0}{ }^{\prime}\left(k_{r s}^{r} r\right)\end{array}\right] ; \quad H_{0}^{\prime}(\chi)=\frac{\partial}{\partial \chi} H_{0}(\chi)$.

According to Hook's law, the relationship between stress and strain in the surrounding medium is

$$
\left\{\begin{array}{l}
\tilde{\sigma}_{r x}=\mu_{m}\left(\frac{\partial u_{r}}{\partial x}+\frac{\partial u_{x}}{\partial r}\right) \\
\tilde{\sigma}_{r r}=\lambda_{m} \Delta+2 \mu_{m} \frac{\partial u_{r}}{\partial r}
\end{array}\right.
$$

Substituting Eq. (11) into Eq. (12) gives

$$
\left(\begin{array}{c}
\tilde{\sigma}_{r x} \\
\tilde{\sigma}_{r r}
\end{array}\right)=\mathbf{T}_{2}\left(\begin{array}{l}
A_{s} \\
B_{s}
\end{array}\right) \mathrm{e}^{\mathrm{i}\left(\omega t-k_{s} x\right)}
$$

Where $\mathbf{T}_{2}=\left[\begin{array}{cc}-2 \mathrm{i} \mu_{m} k_{s} k_{d s}^{r} \mathrm{H}_{0}{ }^{\prime}\left(k_{d s}^{r} r\right) & \mathrm{i} \mu_{m}\left(2 k_{s}^{2}-k_{r}^{2}\right) \mathrm{H}_{1}\left(k_{r s}^{r} r\right) \\ 2 \mu_{m}\left(k_{d s}^{r}\right)^{2} \mathrm{H}_{0}{ }^{\prime \prime}\left(k_{d s}^{r} r\right)-\lambda_{m} k_{d}^{2} \mathrm{H}_{0}\left(k_{d s}^{r} r\right) & -2 \mu_{m} k_{s} k_{r s}^{r} \mathrm{H}_{1}\left(k_{r s}^{r} r\right)\end{array}\right] ; \quad H_{0}^{\prime \prime}(\chi)=\frac{\partial^{2}}{\partial \chi^{2}} H_{0}(\chi)$. 
Combining Eqs. (10) and (12) and eliminating the potential coefficients $A_{m}, B_{m}$, the relationship between stress and displacement can be expressed by

$$
\left(\begin{array}{c}
\tilde{\sigma}_{r x} \\
\tilde{\sigma}_{r r}
\end{array}\right)=\mathbf{T}\left(\begin{array}{l}
u_{x} \\
u_{r}
\end{array}\right)
$$

Where $\mathbf{T}=\mathbf{T}_{2} \mathbf{T}_{1}^{-1}$

\subsection{Coupling motion equations of pipe-medium}

When single axisymmetric waves are considered, the load distribution at the pipe-medium interface is shown in Fig. 2. It assumes that the pipe and medium are in constant contact during the course of movement, the radial displacement of the surrounding medium at the pipe interface is assumed the same as that of the pipe-wall, $u_{r}=w$. The contact stress of pipe and medium in the radial direction are considered as identical, which expressed as $\tilde{\sigma}_{r r}$. The frictional stress along the pipe surface equals the shear stress in the axial direction, which expressed as $\tau_{x}$. The coupled motion equations given by Eq. (1) can be written as

$$
\left[\begin{array}{ll}
A_{11} & A_{13} \\
A_{31} & A_{33}
\end{array}\right]\left(\begin{array}{l}
u \\
w
\end{array}\right)=\frac{1-v_{p}^{2}}{E h}\left(\begin{array}{c}
-\tau_{x} \\
p_{f}(a)+\tilde{\sigma}_{r r}(a)
\end{array}\right)
$$

According to Donnell-Mushtari shell equation [23], the displacement of pipe can be expressed

$$
\left\{\begin{array}{l}
u=U_{s} \mathrm{e}^{\mathrm{i}\left(\omega t-k_{n s} x\right)} \\
w=W_{s} \mathrm{e}^{\mathrm{i}\left(\omega t-k_{n s} x\right)}
\end{array}\right.
$$

Substituting Eq. (16) into Eq. (15) gives

$$
\left[\begin{array}{cc}
\Omega^{2}-\left(k_{s} a\right)^{2} & -\mathrm{i} v_{p}\left(k_{s} a\right) \\
-\mathrm{i} v_{p}\left(k_{s} a\right) & 1-\Omega^{2}+\delta^{2}\left(k_{s} a\right)^{4}
\end{array}\right]\left(\begin{array}{l}
u \\
w
\end{array}\right)=\frac{1-v_{p}^{2}}{E_{p} h} a^{2}\left(\begin{array}{c}
-\tau_{x} \\
p_{f}(a)+\tilde{\sigma}_{r r}(a)
\end{array}\right)
$$

Where $\Omega$ is the non-dimensional frequency, $\Omega=\omega a / c_{L}=k_{L} a ; c_{L}$ is the shell compressional wave speed; $k_{L}$ is the shell compressional wavenumber, $k_{L}^{2}=\omega^{2} \rho_{p}\left(1-v_{p}^{2}\right) / E_{p}$. For a thin-walled pipe $h / a \square 1$, so $\delta^{2}\left(k_{s} a\right)^{4}$ is very small to be ignored. Then, the displacement of the shell can be solved by the pressure in the pipe $p_{f}(a)$, the shear force between the pipe and the soil $\tau_{x}$ and the compressive stress between the pipe and the soil $\tilde{\sigma}_{r r}$.

According to reference [6], for the liquid cannot withstand shear force, the liquid pressure inside the pipe can be expressed directly as the normal displacement of the pipe wall.

$$
p_{f}(a)=\frac{\omega^{2} \rho_{f} J_{0}\left(k_{f s}^{r} a\right)}{k_{f s}^{r} J_{0}^{\prime}\left(k_{f s}^{r} a\right)} w
$$

Where, $k_{f s}^{r}$ is the internal fluid radial wavenumber which can be expressed $\left(k_{f s}^{r}\right)^{2}=k_{f}{ }^{2}-k_{s}{ }^{2}$, here, $k_{f}=\omega / c_{f}$ is the 
fluid wavenumber and $c_{f}=\sqrt{B_{f} / \rho_{f}}$ is the free-field fluid wavespeed; $B_{f}$ is the bulk modulus and $\rho_{f}$ is the density of the internal fluid; $J_{0}$ is a Bessel function of order zero; $J_{0}{ }^{\prime}=(\partial / \partial \chi) J_{0}(\chi)$.

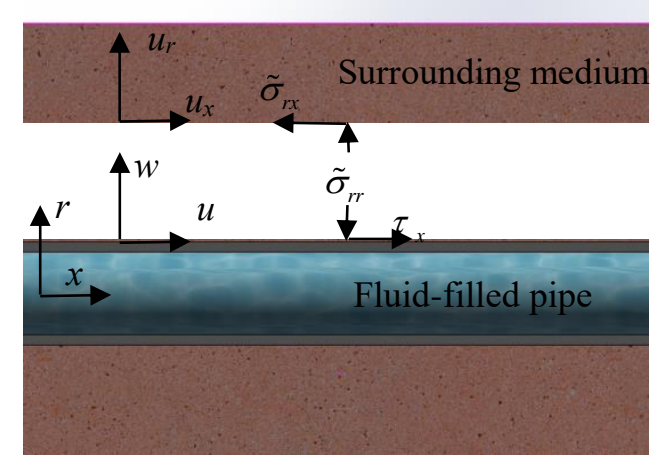

Fig. 2 Displacements and stresses acting at the pipe soil interface.

It is challenging to give a more realistic coupling equation between soil and pipeline because of the complexity of soil properties. Pipeline and soil are usually defined as two extreme conditions, lubrication contact and compact contact to solve the wavenumber [18]. The actual boundary at pipe-soil interface is not both but a transient state. In recent studies, contact coefficients $\xi \in[0,1]$ have been used to characterize this uncertain coupling relationship. $\xi=1$ represents compact contact and $\xi=0$ represents lubrication contact, then at the pipe soil interface $\xi u=u_{x} \quad w=u_{r}$. The force at the interface of the pipe and soil shown by Eq. (14) may be expressed separately by the displacement of the pipe,

$$
\left\{\begin{array}{l}
\tau_{x}=T_{11} \xi u+T_{12} w \\
\tilde{\sigma}_{r r}(a)=T_{21} \xi u+T_{22} w
\end{array}\right.
$$

Substituting Eqs. (18) and (19) into Eq. (17) to eliminate the sine term $\mathrm{e}^{\mathrm{i}\left(\omega t-k_{s} x\right)}$, The axisymmetric $s$ wave coupled equations of motion can be obtained for the buried fluid-filled pipe, as

$$
\left\{\begin{array}{c}
{\left[\Omega^{2}-\left(k_{s} a\right)^{2}+\frac{1-v_{p}^{2}}{E_{p}} \frac{a^{2}}{h} T_{11} \xi\right] U_{s}-\left[\mathrm{i} v_{p}\left(k_{s} a\right)-\frac{1-v_{p}^{2}}{E_{p}} \frac{a^{2}}{h} T_{12}\right] W_{s}=0} \\
{\left[-\mathrm{i} v_{p}\left(k_{s} a\right)-\frac{1-v_{p}^{2}}{E_{p}} \frac{a^{2}}{h} T_{21} \xi\right] U_{s}+\left[1-\Omega^{2}+\delta^{2}\left(k_{s} a\right)^{4}-\frac{\rho_{f}}{\rho_{p}} \frac{a}{h} \frac{\Omega^{2}}{k_{f s}^{r} a} \frac{J_{0}\left(k_{f s}^{r} a\right)}{J_{0}^{\prime}\left(k_{f s}^{r} a\right)}-\frac{1-v_{p}^{2}}{E_{p}} \frac{a^{2}}{h} T_{22}\right] W_{s}=0}
\end{array}\right.
$$

Solving Eq. (20) can be obtained

$$
\begin{aligned}
& {\left[\Omega^{2}-\left(k_{s} a\right)^{2}+\frac{1-v_{p}^{2}}{E_{p}} \frac{a^{2}}{h} T_{11} \xi\right]\left[1-\Omega^{2}-\frac{\rho_{f}}{\rho_{p}} \frac{a}{h} \frac{\Omega^{2}}{k_{f s}^{r} a} \frac{J_{0}\left(k_{f s}^{r} a\right)}{J_{0}^{\prime}\left(k_{f s}^{r} a\right)}-\frac{1-v_{p}^{2}}{E_{p}} \frac{a^{2}}{h} T_{22}\right]} \\
& =\left[\mathrm{i} v_{p}\left(k_{s} a\right)-\frac{1-v_{p}^{2}}{E_{p}} \frac{a^{2}}{h} T_{12}\right]\left[\mathrm{i} v_{p}\left(k_{s} a\right)+\frac{1-v_{p}^{2}}{E_{p}} \frac{a^{2}}{h} T_{21} \xi\right]
\end{aligned}
$$

The fluid loading term, $F L$ [7] and the surrounding medium loading matrix, SL, are given by 


$$
\begin{gathered}
F L=\frac{\rho_{f}}{\rho_{p}} \frac{a}{h} \frac{\Omega^{2}}{k_{f s}^{r} a} \frac{J_{0}\left(k_{f s}^{r} a\right)}{J_{0}^{\prime}\left(k_{f s}^{r} a\right)} \\
\mathbf{S L}=\frac{\left(1-v_{p}^{2}\right)}{E_{p}} \frac{a^{2}}{h} \mathbf{T}
\end{gathered}
$$

then Eq. (21) can be written in a simple form

$$
\left[\Omega^{2}-\left(k_{s} a\right)^{2}+\xi S L_{11}\right]\left[1-\Omega^{2}-F L-S L_{22}\right]=\left[\mathrm{i} v_{p}\left(k_{s} a\right)-S L_{12}\right]\left[\mathrm{i} v_{p}\left(k_{s} a\right)+\xi S L_{21}\right]
$$

\section{Wave characteristic}

For the $\mathrm{s}=1$ wavenumber in buried fluid-filled pipes, $k_{1}^{2} \square k_{L}^{2}$, so $\left(k_{1} a\right)^{2} \square \Omega^{2}$. When the frequency is low, $k_{f s}^{r} \rightarrow 0$. According to the properties of Bessel functions, $\chi \rightarrow 0, \frac{J_{0}(\chi)}{J_{0}^{\prime}(\chi)} \approx-\frac{2}{\chi}$. Then the fluid loading term, $F L$, can be simplified as

$$
F L=-2 \frac{\rho_{f}}{\rho_{p}} \frac{a}{h} \frac{\Omega^{2}}{\left(k_{f}^{2}-k_{s}^{2}\right) a^{2}}
$$

Substituting Eq. (25) into Eq. (24) gives

$$
k_{1}^{2}=k_{f}^{2}\left(1+\frac{\beta}{1-\Omega^{2}+\alpha}\right)
$$

where,

$$
\left\{\begin{array}{l}
\alpha=-S L_{22}-\frac{\left[v_{p}+\mathrm{i} S L_{12} / k_{1} a\right]\left[v_{p}-\mathrm{i} \xi S L_{21} / k_{1} a\right]}{1-\xi S L_{11} / k_{1} a} \\
\beta=2 \frac{a}{h}\left(\frac{1-v_{p}^{2}}{E_{P}}\right) B_{f}
\end{array}\right.
$$

where $\alpha$ stands for the surrounding medium loading and pipe parameters which can be used to evaluate the influence of soil load on the pipe wall displacement. $\beta$ refers to fluid and pipe parameters which can be used to evaluate the influence of fluid load on the pipe wall displacement. By means of a complex modulus of elasticity $E p$ ( $\alpha$ and $\beta$ always complex), it is found from Eq. (26) that $k_{l}$ is always complex indicating the $s=1$ wave decays as it propagates. Then $\alpha$ and $\beta$ are described as the measures of the loading effects of surrounding medium and fluid on the pipe wall respectively.

By Eq. (27), $\beta$ can be obtained directly, but $\alpha$ which is related to the unknown wavenumber $k_{l}$ cannot be solved directly. When the pipe is placed in a different medium, the equations can be simplified by boundary conditions.

\subsection{Lubrication Contact of Pipe-Medium}

On the condition of lubrication contact, the contact coefficient $\xi=0$, the measure of the loading effects of the 
surrounding medium $\alpha=-v_{p}^{2}-S L_{22}-\mathrm{i} v_{p} S L_{12} / k_{1} a$, then the Eq. (24) can be written as

$$
k_{1}^{2}=k_{f}^{2}\left(1+\frac{\beta}{1-\Omega^{2}-v_{p}^{2}-S L_{22}-\mathrm{i} v_{p} S L_{12} / k_{1} a}\right)
$$

It can be seen from Eq. (28) that the propagation of $k_{l}$ wave will be delayed as it propagates caused by the effect of the pipe wall (i.e., a complex $\beta$ ) and additional damping of the surrounding medium (i.e., a complex $\alpha$ ), although there is no frictional damping between pipe and surrounding medium.

\subsection{Pipe in Non-viscous liquids}

For non-viscous liquids, the shear modulus $\mu_{m}=0$, contact coefficient $\xi=0$, as a result, the Lame coefficient $\lambda_{m}=B_{m}$, the shear wavenumber $k_{r} \rightarrow \infty$, and $\mu_{m} k_{r}^{2}=\omega^{2} \rho_{m}$. Then the surrounding medium loading matrix SL can be simplified as $S L_{11}=S L_{12}=0$. In this case, the measure of the loading effects of the surrounding fluid reduces to $\alpha=-v_{p}^{2}-S L_{22}$. Eq. (24) can be rewritten as

$$
k_{1}^{2}=k_{f}^{2}\left(1+\frac{\beta}{1-\Omega^{2}-v_{p}^{2}-S L_{22}}\right)
$$

Where, $S L_{22}=-\frac{\rho_{m}}{\rho_{p}} \frac{a^{2}}{h} \frac{k_{L}^{2}}{k_{d 1}^{r}} \frac{H_{0}\left(k_{d 1}^{r} a\right)}{H_{0}^{\prime}\left(k_{d 1}^{r} a\right)}$. Since $S L_{22}$ is a function of the complex $k_{l}, \alpha$ is a complex value. So, $s_{l}$ wave attenuation is attributed to both material losses along the pipe wall (i.e., a complex $\beta$ ) and radiation losses due to the added damping of the surrounding medium (i.e., a complex $\alpha$ ).

\subsection{Pie in Air}

For an air medium, the loading effects of air on the pipe wall can be neglected, the contact coefficient is considered zero. Then, $\mathbf{T}=0, \mathbf{S L}=0$, and $\alpha=-v_{p}^{2}$. Eq. (24) can be expressed are consistent with the reference [9].

$$
k_{1}^{2}=k_{f}^{2}\left(1+\frac{\beta}{1-\Omega^{2}-v_{p}^{2}}\right)
$$

At low frequency, $\Omega^{2} \square 1$, and $\operatorname{Re}(\beta) \square 1$, then $k_{1}^{2}>k_{f}^{2}$. And that means the wave speed of the $s=1$ wave will be significantly lower than that of the free wave. In Eq. (30), the imaginary part only exists in $\beta$, so the wave attenuation is only due to losses within the pipe wall.

Compared to the equation from Eq. (26) to Eq. (30), it can be seen that, if the real part of $\alpha$ less than zero, $\operatorname{Re}(\alpha)<0$, external loads of surrounding medium act as additional mass, and the wavenumber will increase compared to the in-air value; Contrarily, if the real part of $\alpha$ more than zero, $\operatorname{Re}(\alpha)>0$, external loads of surrounding medium act as additional damping, and the wavenumber will decrease relative to the in-air case. 


\section{Numerical results and discussions}

4.1 Numerical method

This section presents some numerical results of the shear effect on $s=1$ wavenumber. The real part $k_{r e}$ and imaginary part $k_{i m}$ of $k_{l}$ are set as variables and the Eq. (24) is described as a target function

$$
F(k r, k i)=\left|\left[\Omega^{2}-\left(k_{s} a\right)^{2}-S L_{11}\right]\left[1-\Omega^{2}-F L-S L_{22}\right]-\left[\mathrm{i} v_{p}\left(k_{s} a\right)+S L_{12}\right]^{2}\right| \rightarrow \min
$$

The Neldes-Mead method[24] is used to solve Eq. (31). In the optimization progress, the termination condition is set as

$$
\left\{\frac{1}{3} \sum_{i=1}^{3}\left[F\left(k_{r e}^{(n)}, k_{i m}^{(n)}\right)-F\left(\bar{k}_{r e}, \bar{k}_{i m}\right)\right]^{2}\right\}^{1 / 2}<\varepsilon
$$

Where, $n$ is the iterations, $\bar{k}_{r e}, \bar{k}_{i m}$ are the centre of the simplex in the current step, $\varepsilon$ is the tolerance.

In the calculation process, the derivative of Bessel function can be deal based on its property.

$$
\left\{\begin{array}{l}
J_{0}{ }^{\prime}\left(k_{f s}^{r} a\right)=-J_{1}\left(k_{f s}^{r} a\right) \\
H_{0}{ }^{\prime}\left(k_{d s}^{r} a\right)=-H_{1}\left(k_{d s}^{r} a\right) \\
H_{0}{ }^{\prime}\left(k_{d s}^{r} a\right)=\left(H_{2}\left(k_{d s}^{r} a\right)-H_{0}\left(k_{d s}^{r} a\right)\right) / 2 \\
H_{1}{ }^{\prime}\left(k_{r s}^{r} a\right)=\left(-H_{2}\left(k_{r s}^{r} a\right)+H_{0}\left(k_{r s}^{r} a\right)\right) / 2
\end{array}\right.
$$

For the arguments of the Bessel or Hankel functions are derived from the square root, it is important to choose the sign of the root. The method to choose the sign of the root in reference [18] is be used. If the real part is larger, the partial wave can be considered homogeneous and must propagate away from the shell, so the positive square root is chosen. On the other hand, if the imaginary part is larger, the partial wave can be considered inhomogeneous and must decay away from the shell, so the negative square root is selected.

The material properties of the fluid, pipe and surrounding medium are shown in Table 1. Considering the efficiency and convergence, the wavenumbers are calculated up to $1 \mathrm{~Hz}$. The thickness/radius ratio of pipe is 0.125 , and the plate compressional wave speed is $1725 \mathrm{~m} / \mathrm{s}$.

Table 1 Properties of the fluid, water and surrounding medium

\begin{tabular}{llll}
\hline Properties & Fluid & Pipe & Surrounding medium \\
\hline Density $\left(\mathrm{kg} / \mathrm{m}^{3}\right)$ & 1000 & 2000 & 2000 \\
Young's modulus $\left(\mathrm{N} / \mathrm{m}^{2}\right)$ & -- & $5.0 \times 10^{9}$ & -- \\
Bulk modulus $\left(\mathrm{N} / \mathrm{m}^{2}\right)$ & $2.25 \times 10^{9}$ & -- & $4.5 \times 10^{9}$ \\
Shear modulus $\left(\mathrm{N} / \mathrm{m}^{2}\right)$ & -- & -- & $2 \times 10^{7} \sim 10 \times 10^{7}$ \\
Poisson's ratio & -- & 0.4 & -- \\
Material loss factor & -- & 0.065 & -- \\
\hline
\end{tabular}


Considering the previous analysis, the shear effect of surrounding medium and shear effect of pipe-medium interface both affect the wave propagation and attenuation characteristics. This section presents some numerical sample to discuss the influence of the shear effects. The attenuations are defined by the loss in $\mathrm{dB}$ per unit propagation distance by

$$
\operatorname{Loss}(\mathrm{dB} / \mathrm{unit} \text { distance a })=-20 \frac{\operatorname{Im}\left\{k_{1} a\right\}}{\ln (10)}
$$

\subsection{Shear effect of surrounding medium}

Some numerical results are shown in this section to explain the influence of the shear effect of surrounding medium. In order to eliminate the effects of surrounding medium and pipe interface friction, the "lubrication contact" assume is used here. Fig. 3 gives the wavenumber for the soil with different shear modulus. It can be seen that from Fig.3(a), as the previous theoretical analysis, the effect of the pipe and surrounding medium is used to substantially increase the real part of the $s=1$ wavenumber from the free-field value $k_{f}$. This solution is similar to the reference [18]. As the shear effect of the surrounding medium increased, the real part of the wavenumber gradually decreased. The overall loading influences of the surrounding medium are to add mass to the pipe wall, but the shear effect is to add stiffness, and additional stiffness increases with the increase of the shear effect of surrounding medium.

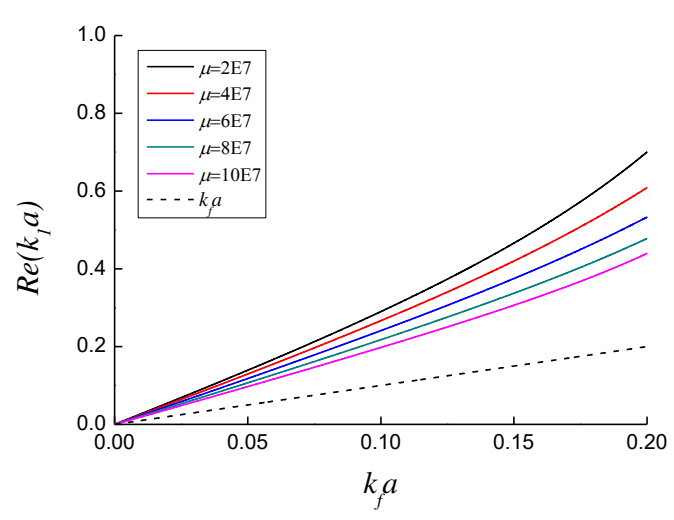

Fig.3 Real part of wavenumber for $s=1$ wave

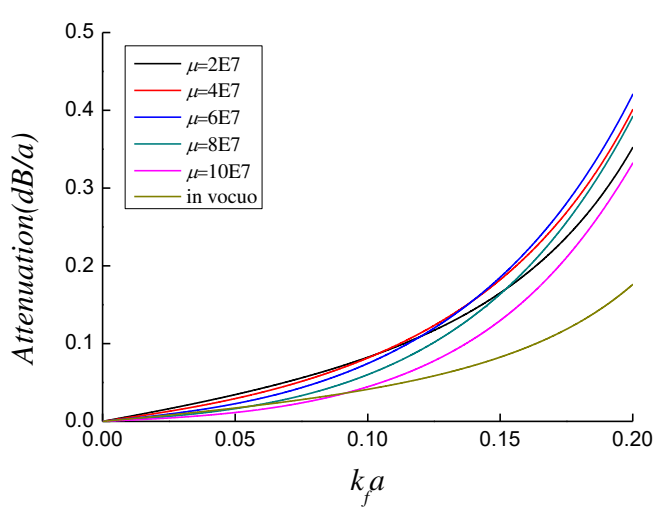

Fig.4 Loss of $s=1$ wave

Fig.4 shows the loss in $\mathrm{dB}$ per unit propagation distance. Compared with the attenuation in vacuo, the radiation into the surrounding medium is significantly used for much larger attenuation. The attenuation is more serious in the high-frequency range. In fact, at the lower frequency range, the attenuation dominated by losses within the pipe, as the shear effects increased, the attenuation slightly decreased. At higher frequencies, radiation as both compressional waves and shear waves contributes to the attenuation, and then the attenuation increased with the shear effects increased. So the shear effects of surrounding medium should not be neglected in leak detection of buried pipeline. 
In order to better understand the shear effects of surrounding medium on dispersive behaviour of $s=1$ wave, the real and imaginary parts of the measure $\alpha$ are given in Fig.5 and Fig.6 respectively. As shown in these figures, the level of $\operatorname{Re}(\alpha)$ is much larger than $\operatorname{Im}(\alpha)$, thus $\operatorname{Re}(\alpha)$ plays a dominant role in the overall loading effects of surrounding medium on the propagation characteristics. As mentioned above, a negative $\operatorname{Re}(\alpha)$ indicates that the surrounding medium is to add mass to the pipe wall and the propagation wavenumber will be increased. With the increase of the shear effects, $\operatorname{Re}(\alpha)$ increased and slightly larger than the value in vacuo, thus the shear effects of surrounding medium have some impact on the propagation characteristics of $s=1$ wave. Additionally, it can be noted that $\operatorname{Re}(\beta) \quad(\operatorname{Re}(\beta)=0.58)$ is much larger than $\operatorname{Re}(\alpha)$, which shows the fluid loading dominants the vibration of the pipe wall for plastic pipe, so more attention should be paid to fluid load wave in leak detection.

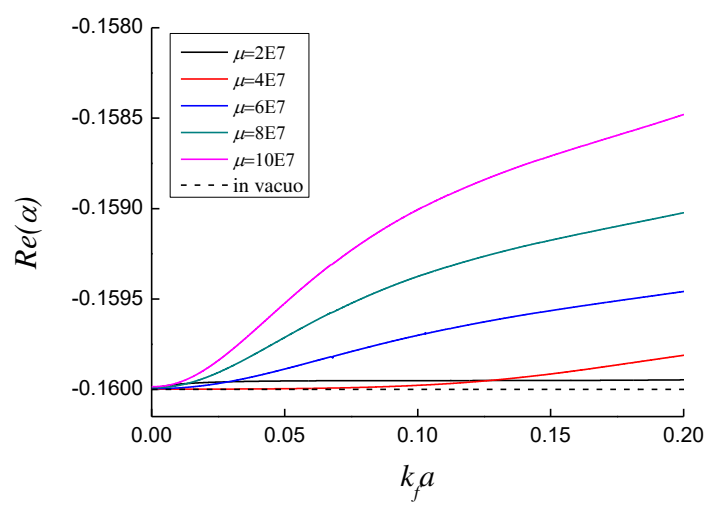

Fig.5 Real part of the measure effect $\alpha$

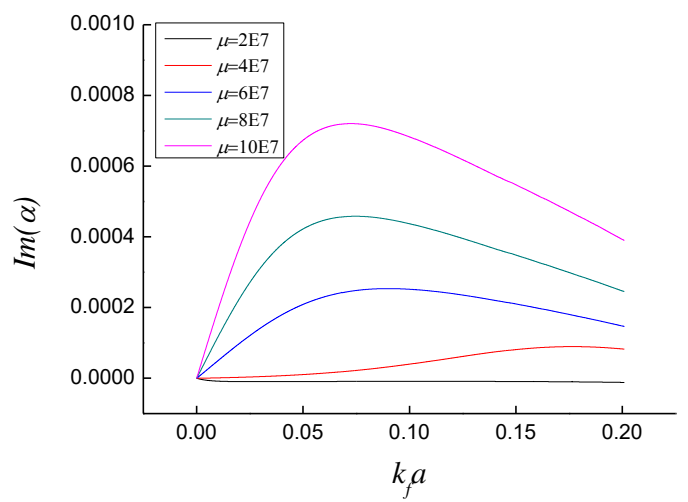

Fig.6 Imaginary part of the measure $\alpha$

\subsection{Shear effects at the pipe/medium interface}

The shear effects at the pipe/medium interface exist in buried pipe systems, but it is normally ignored because the coupling relationship between pipes and surrounding medium is not clear. Fig.7 and Fig. 8 show the wavenumber for $s=1$ wave with different contact coefficients. In calculation, the shear wave speed of surrounding medium is set to $300 \mathrm{~m} / \mathrm{s}$.

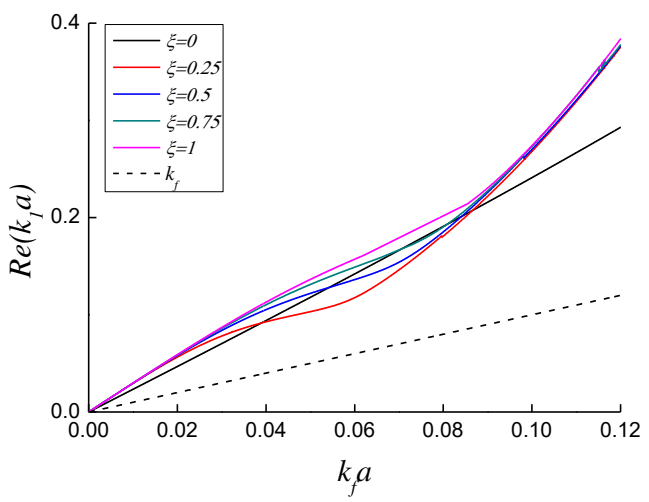

Fig. 7 Real part of wavenumber for $s=1$ wave

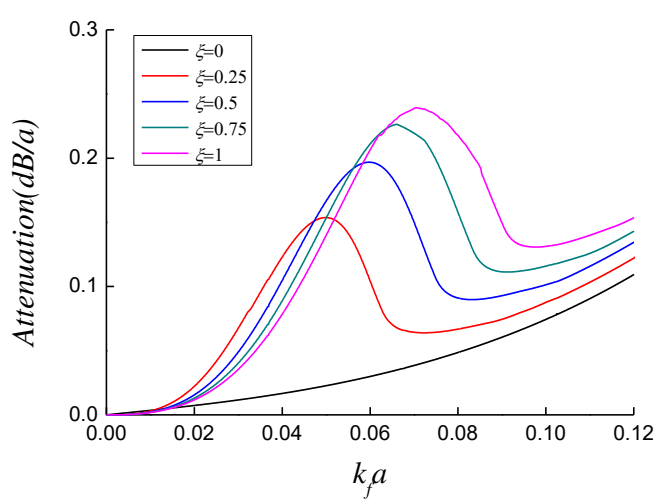

Fig. 8 Loss of $s=1$ wave 
As shown in Fig.7, the real part of wavenumber for $s=1$ wave much larger than the free-field value $k_{f}$ with different contact coefficients. Whether the shear effect of the interface between surrounding medium and pipe wall has a great influence on the real part at high frequency $\left(k_{f} a>0.09\right)$. Once these shear effects are included, the influence of the contact coefficient on the real part is mainly reflected in the middle frequency band $\left(k_{f} a \in(0.02,0.09)\right)$. In Fig.8, it can be seen that with the consideration of the shear effect of the interface, more waves can be radiated into the surrounding medium. The attenuation increased as the frequency increased, and has a local peak in the middle frequency band. The frequency corresponding to these peaks increased with the contact coefficient increased. The local peak of attenuation is caused by the resonance between the additional mass of the surrounding medium and the pipe wall, resulting in a dramatic increase in the wave intensity of radiation to the surrounding medium. At the high frequency, the friction force between surrounding medium and pipe wall seems not enough for surrounding medium to vibrate with the pipe wall, so the results of attenuation gradually approaching the value in the case of lubrication contact.

In engineering applications, the actual wave number and attenuation should be obtained according to the actual pipe-soil coupling situation, and the delay estimation should be carried out according to the leakage signal dispersion in order to obtain the accurate leakage location results. Medium and low-frequency signal is generally be used, and the contact coefficient which has a certain influence on the propagation characteristics of the wave in this frequency band should not be ignored.

\subsection{Field test}

This section presents some numerical results of wavenumber from actual plastics water pipes. Two field tests were carried out for pipes in different buried environments. The details of the experimental setup and analysis for test 1 can be found in Ref. [17, 20]. The test 2 selected the water supply network in Southwest Jiaotong University, the sensor is set in the pipe well, and fire hydrant discharge signal as leakage signal. The distance from pipe well to hydrant discharge is 12 m. The soil and pipe coupling parameters cannot be obtained in both tests, the theoretical calculation and experimental comparison are not presented in this paper.

Fig.9 and Fig.10 show the real and the imaginary components of the measured and fitting wavenumbers. Due to the different buried environments of pipes in the two tests, both the real and imaginary components of the measured wavenumbers are large different. There are some reflections from pipe connections blow $160 \mathrm{~Hz}$ which cause some fluctuations at the corresponding frequency. At the high frequency the measured data becomes unreliable due to the noise

interference. Experimental measurements show good agreement on the trend of the wavenumbers with predictions in Fig.7 and Fig.8. Due to the shear effects at the pipe/medium interface, the attenuations have a local peak (about $700 \mathrm{~Hz}$ 


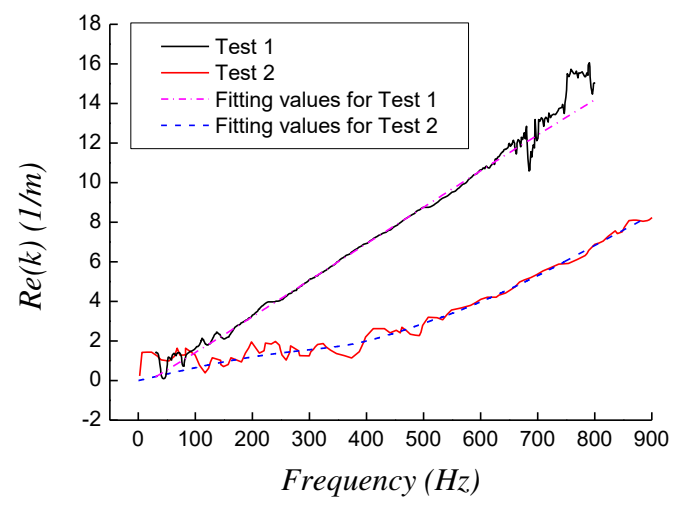

Fig.9 Real part of measured wavenumber

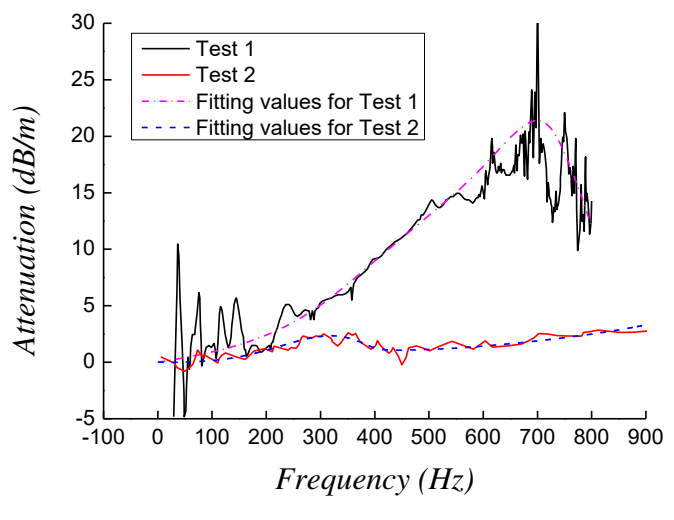

Fig.10 Loss of measured wavenumber

\section{Conclusion}

Axisymmetric waves in thin-walled fluid-filled plastic pipe surrounded by an infinite elastic medium which can sustain both longitudinal and shear waves have been studied. The contact coefficient has been introduced to describe the contact strength of pipe and surrounding medium. Then a general expression for the fluid-dominated wavenumber has been presented in buried fluid-filled plastic pipe.

For axisymmetric waves, the fluid loading dominates the vibration of the plastic pipe wall. The overall loading effects of the surrounding medium are to add mass to the pipe wall, but the shear effect is to add stiffness, which increases with the shear effect of surrounding medium. The shear effect of the surrounding medium also influences the attenuation of the wave.

The added mass of the surrounding medium will resonate with the pipe wall at a specific frequency under the shear effects at the pipe/medium interface, resulting in the change of the propagation characteristics of the wave near the frequency. At higher frequency, the influence of shear effects at the interface on the propagation characteristics is not obvious. The wavenumber can be solved by compact contact theory and the attenuation will approximate the lubricating contact state with the frequency increases.

\section{Declarations}

Availability of data and materials

This paper includes all the data and materials which can be shared. 
Competing interests

Not applicable.

Funding

National Nature Science Foundation of China (No.11774378).

Authors' contributions

Ping Lu completed the equation derivation, calculation and writing of the paper.

Xiaozhen Sheng guided the research ideas and results of the paper.

Yan Gao proposed the research idea of this article.

Ruichen Wang revised the language of the paper.

Acknowledgements

The authors gratefully acknowledge the support of the National Nature Science Foundation of China (No.11774378).

\section{References}

[1] Fuchs Hv, Riehle R. Ten years of experience with leak detection by acoustic signal analysis. Apple Acoust 1991,33:1-19.

[2] Muggleton JM, Brennan MJ, Linford PW. Wavenumber prediction of waves in buried pipes for water leak detection. J Sound Vib 2002, 249:939-954.

[3] Hunaidi O, Wang A. A new system for locating leaks in urban water distribution pipes. J Environ Qual 2006, 7(4):450-66.

[4] Gao Y, Brennan MJ, Joseph PF. A comparison of time delay estimators for the detection of leak noise signals in plastic water distribution pipes. J Sound Vib 2006, 292:552-570.

[5] Brennan MJ, Gao Y, Joseph PF. On the relationship between time and frequency domain methods in time delay estimation for leak detection in water distribution pipes, J Sound Vib 2007,304:213-223.

[6] Fuller CR, Fahy FJ. Characteristics of wave-propagation and energy distributions in cylindrical elastic shells filled with fluid. J Sound Vib 1982;81:501-18.

[7] Fuller CR. The input mobility of an infinite circular cylindrical elastic shell filled with fluid. J Sound Vib 1983;87:409-27.

[8] Xu MB, Zhang WH. Vibrational power flow input and transmission in a circular cylindrical shell filled with fluid. J Sound Vib 2000;234: 387-403. 
[9] Pinnington RJ, Briscoe AR. Externally applied sensor for axisymmetric waves in fluid-filled pipe. J Sound Vib 1994,173(4):503-516.

[10] Pinnington RJ. The axisymmetric wave transmission properties of pressurized flexible tubes. J Sound Vib 1997;204:271-89.

[11] Pinnington RJ. Axisymmetric wave transfer functions of flexible tubes. J Sound Vib 1997;204:291-310.

[12] BikashK. Sinha, ThomasJ. Plona, SergioKostek, ShuKongChang, Axisymmetric wave propagation in fluidloaded cylindrical shells. I:theory, Journal of the Acoustical Society of America 92 (1992)1132.

[13] JoshuaE. Greenspon, Vibrations of thick and thin cylindrical shells surrounded by water, Journal of the Acoustical Society of America 33 (1961)1321.

[14] Long R, Cawley P, Lowe MJS. Acoustic wave propagation in buried iron water pipes. Proc Royal Soc Lond: Mathematical, Physical and Engineering Sciences 2003,459:2749-2770.

[15] Zhang J, Jia L, Shu Y, Wave propagation characteristics of the shells of revolution by frequency wave number spectrum method. Journal of Sound and Vibration. 2002.251(2):367-372.

[16]Muggleton JM, Brennan MJ, Pinnington RJ. Wavenumber prediction of waves in buried pipes for water leak detection. J Sound Vib 2002;249:939-54.

[17] Muggleton JM, Brennan MJ, Linford PW. Axisymmetric wave propagation in fluid-filled pipes: wavenumber measurements in in vacuo and buried pipes. J Sound Vib 2004;270:171-90.

[18] Muggleton JM, Yan J. Wavenumber prediction and measurement of axisymmetric waves in buried fluid-filled pipes: inclusion of shear coupling at a lubricated pipe/soil interface. J Sound Vib 2013;332:1216-30.

[19] Gao Y, Sui F, Muggleton JM, Yang J. Simplified dispersion relationships for fluid-dominated axisymmetric wave motion in buried fluid-filled pipes. J Sound Vib 2016;375:386-402.

[20] Gao Yan, Liu Yuyou, Jennifer M. Muggleton. Axisymmetric fluid-dominated wave in fluid-filled plastic pipes: Loading effects of surrounding elastic medium. Applied Acoustics 2017,116:43-49.

[21] A.W. Leissa, Vibration of Shells, NASA SSP-288, US Government Printing Office, Washington, DC, 1973.

[22] W.M. Ewing, W.S. Jardetzky, F. Press, Elastic Waves in Layered Media, McGraw-Hill, New York, 1957.

[23] M. C. JUNGER and D. FEIT Sound, Structures and Their Interaction. Cambridge, press 1972

[24] XUE Biyi, Cui Dabin, Li Li, Du Xing, Wen Zefeng, Jin Xuesong. Parallel inverse design method of wheel profile. Journal of Mechanical Engineering, 2013, 49(16):8-16. 
Figures

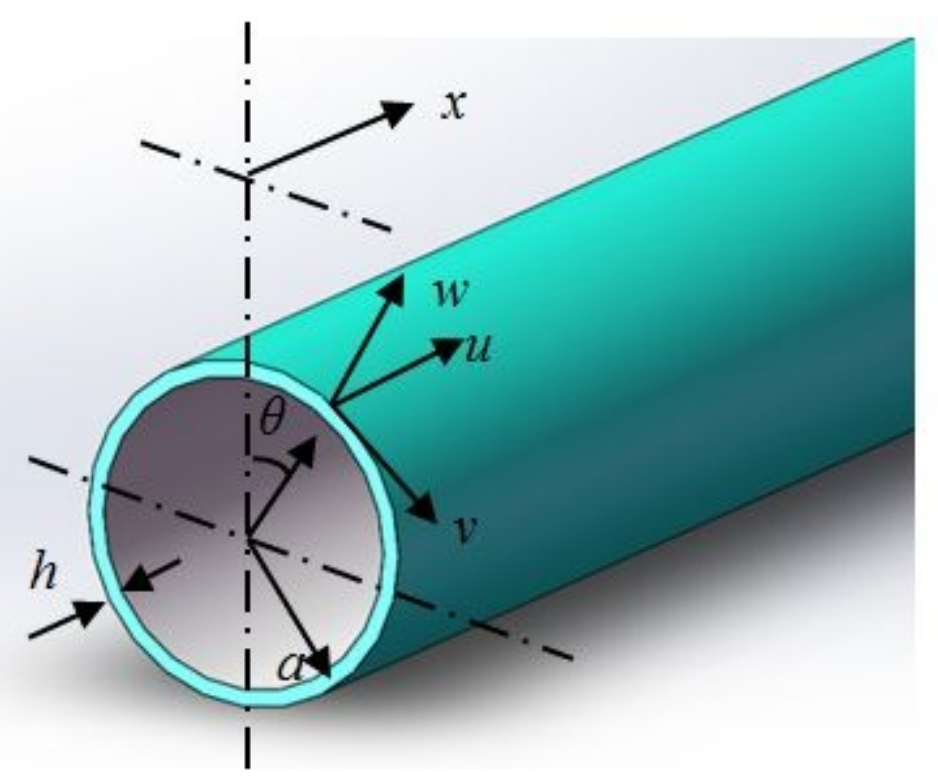

Figure 1

The coordinate system for a buried fluid-filled pipe

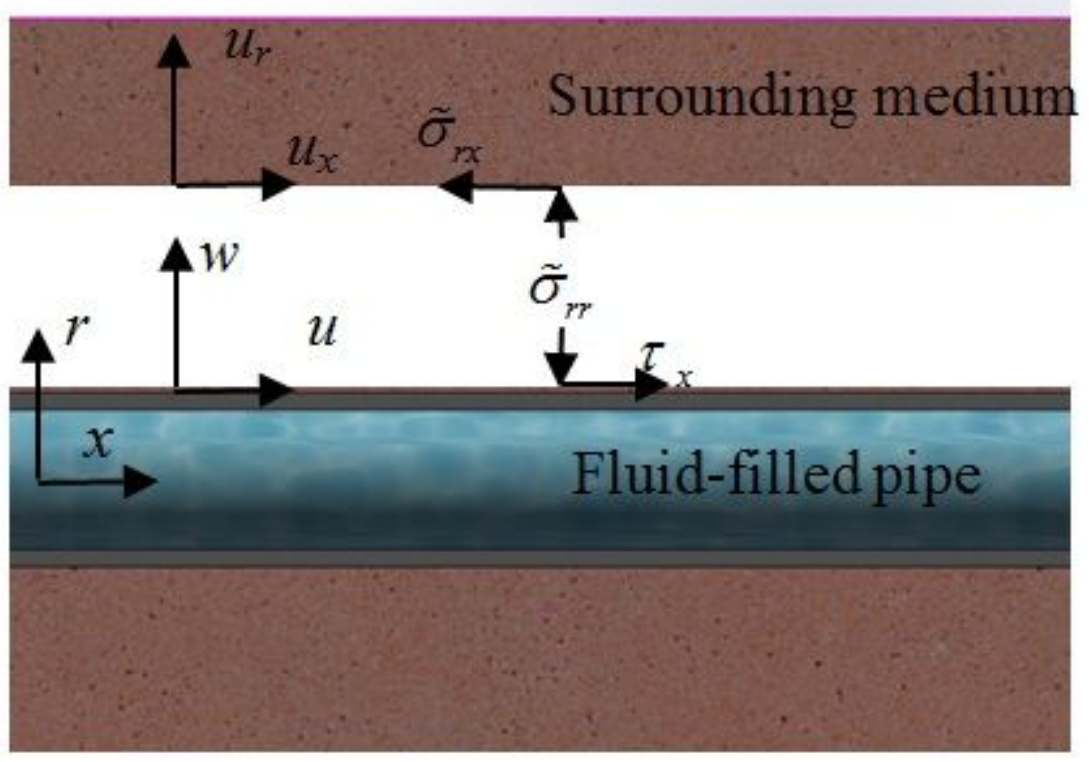

Figure 2

Displacements and stresses acting at the pipe soil interface. 


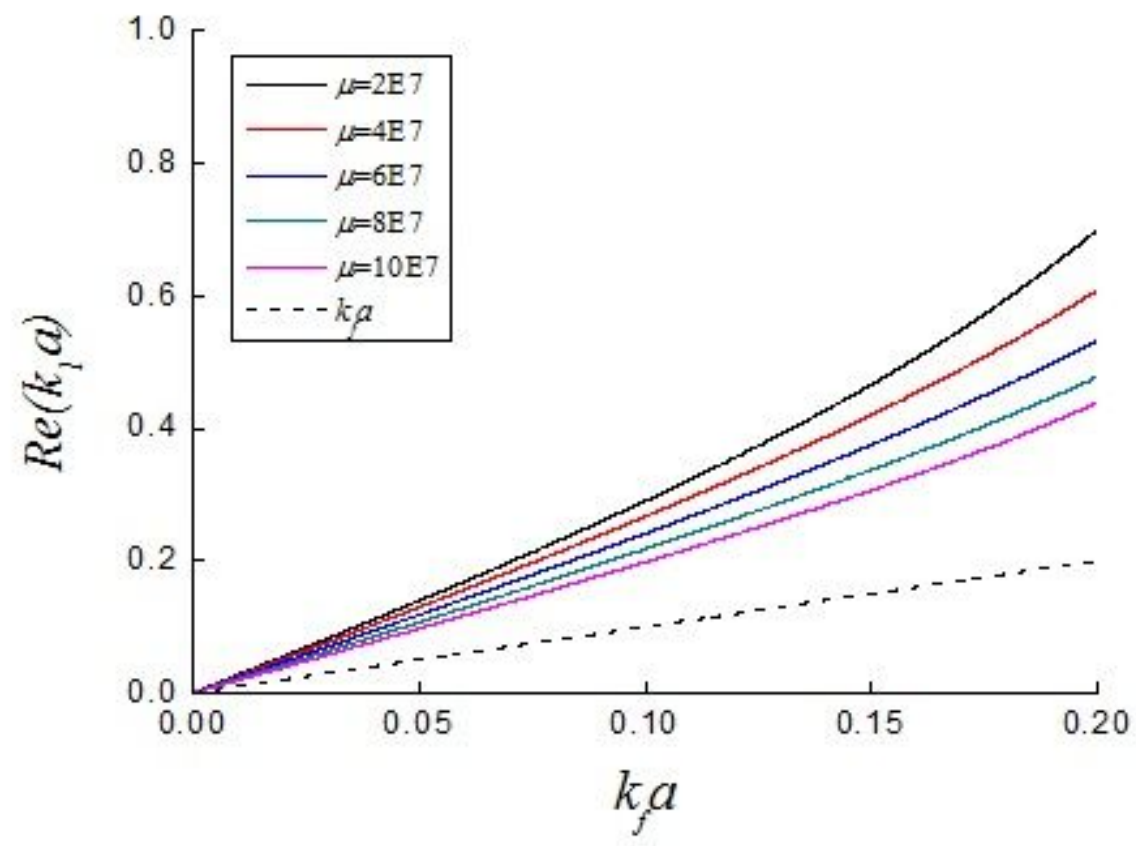

Figure 3

Real part of wavenumber for $s=1$ wave

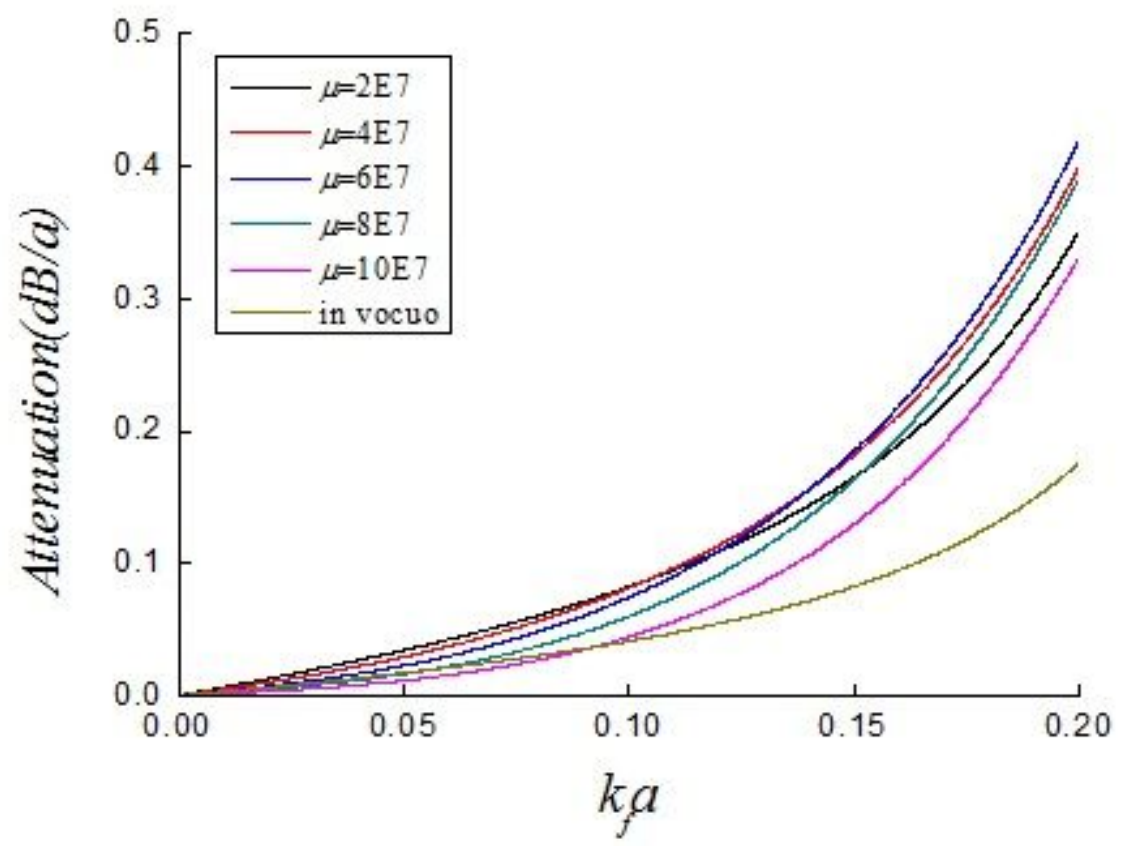

Figure 4

Loss of $s=1$ wave 


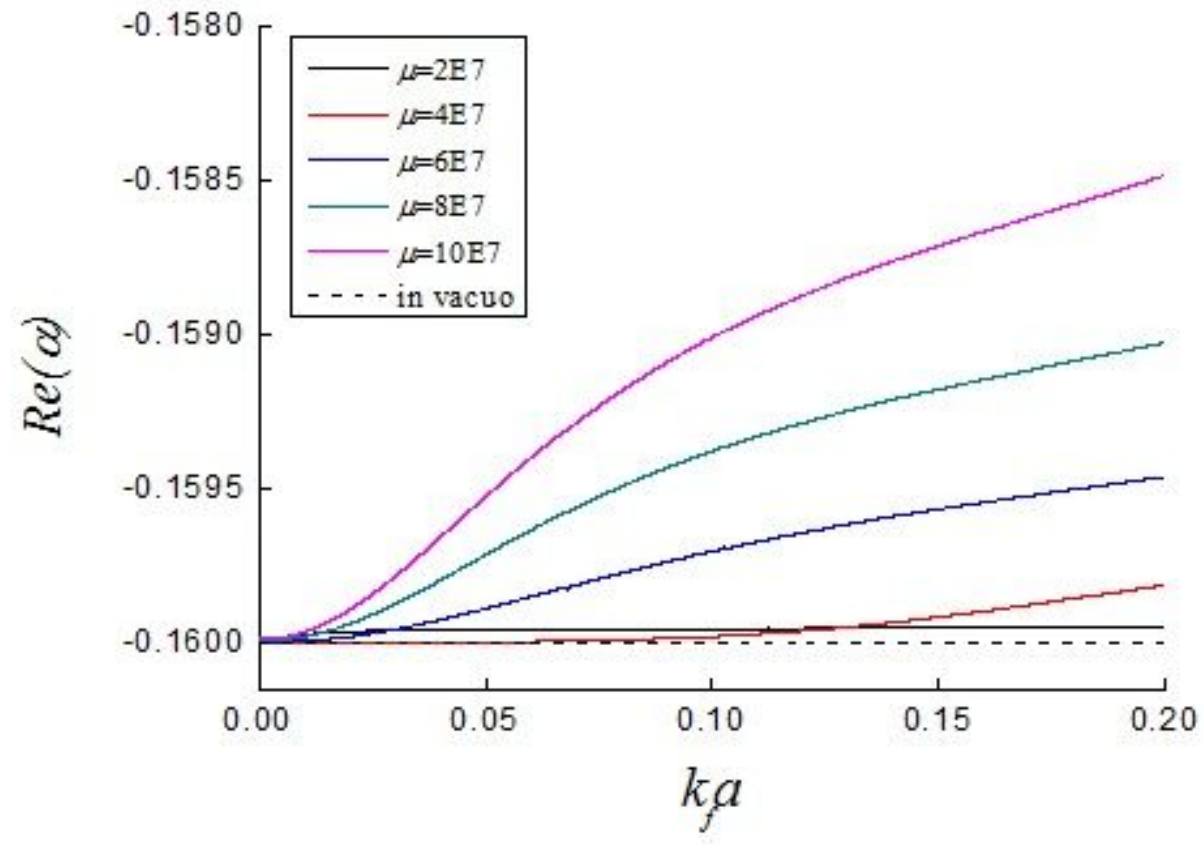

Figure 5

Real part of the measure effect a

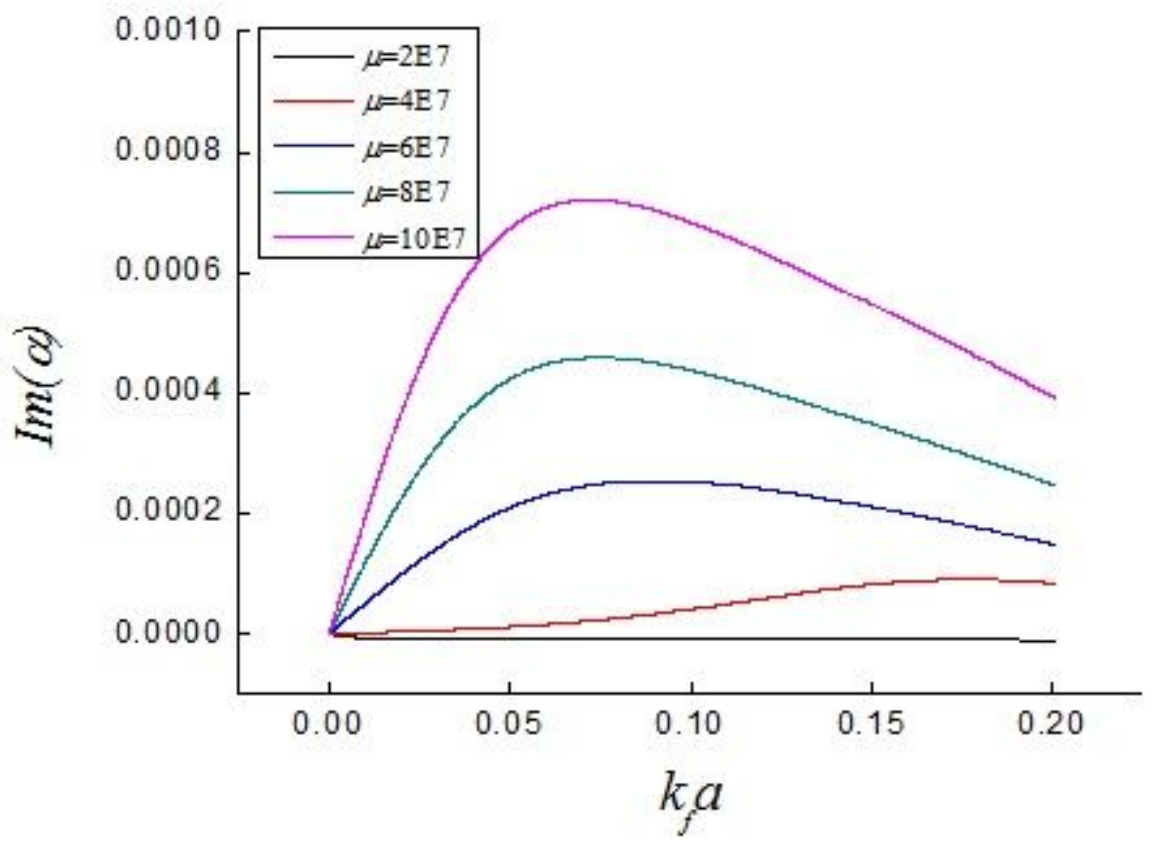

Figure 6

Imaginary part of the measure a 


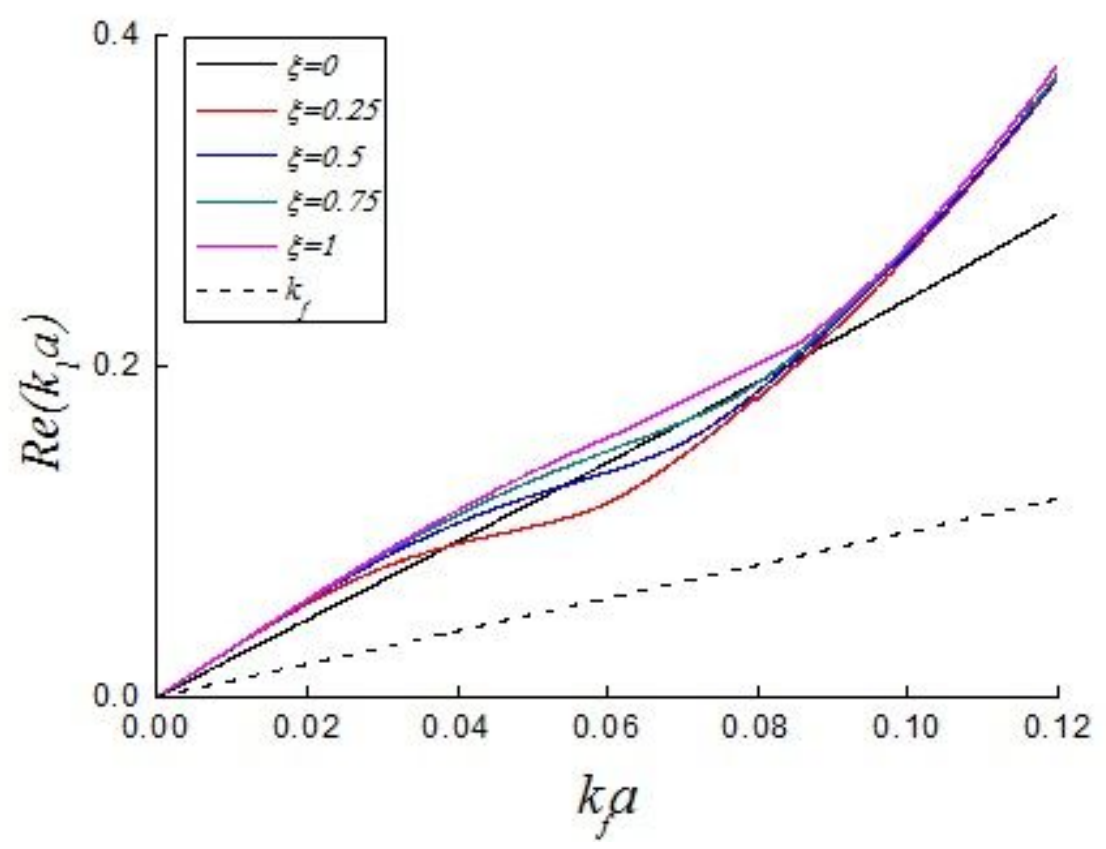

Figure 7

Real part of wavenumber for $s=1$ wave

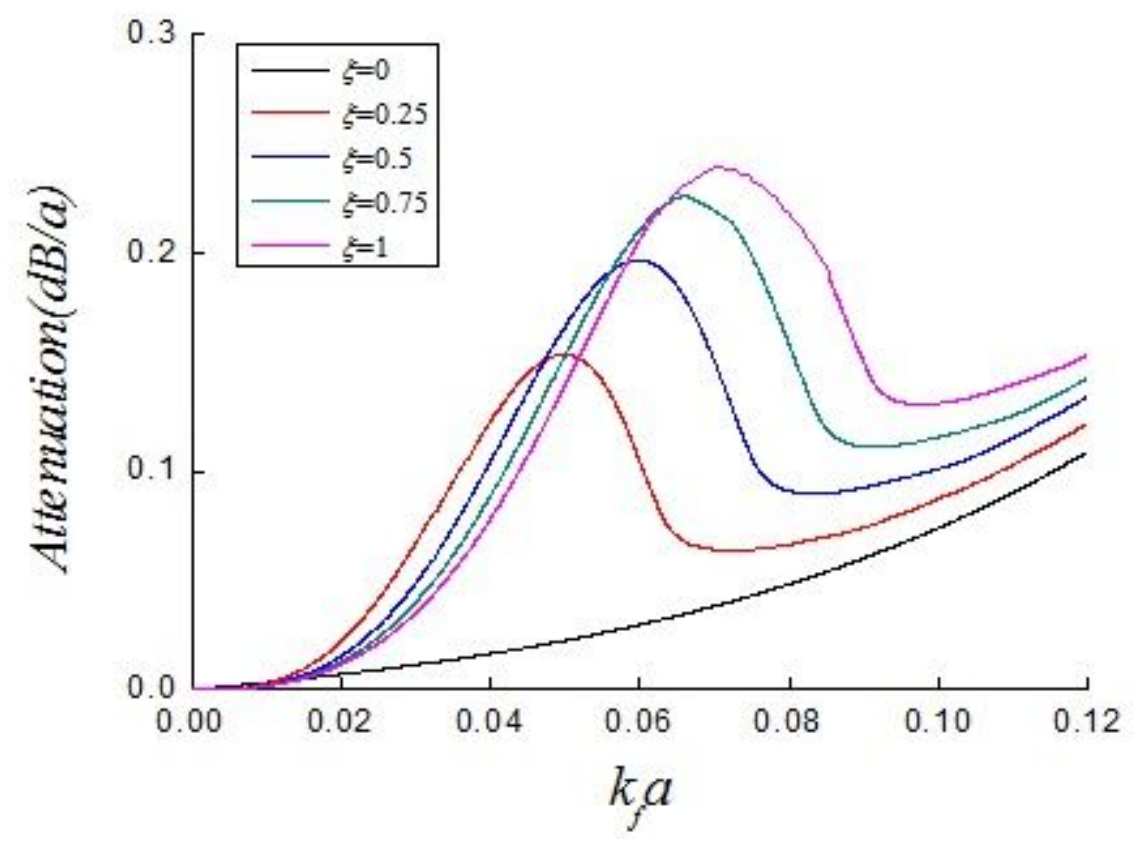

Figure 8

Loss of $s=1$ wave 